\title{
Evaluación de habilidades transversales de comunicación y colaboración a través de un examen clínico objetivo es- tructurado de radiología de urgencias para residentes de un programa universitario de especialidad en radiología.
}

Francisco Garrido C. ${ }^{1,2}{ }^{*}$, Ximena Triviño B. ${ }^{3}$, Héctor Henríquez L. ${ }^{4}$, Pablo Cikutovic M. ${ }^{1}$, Raúl Arau U.5 Álvaro Huete G. ${ }^{1}$

1. Departamento de Radiología y Diagnóstico por Imágenes, Escuela de Medicina, Pontificia Universidad Católica de Chile. Santiago-Chile.

2. Centro de Educación Médica y Ciencias de la Salud, Facultad de Medicina, Pontificia Universidad Católica de Chile. Santiago-Chile.

3. Dirección Académica de Docencia en Salud, Universidad San Sebastián. Santiago - Chile.

4. Departamento de Radiología, Clínica Santa María. Santiago - Chile.

5. Unidad Docente Asociada Complejo Asistencial Dr. Sótero del Río, Pontificia Universidad Católica de Chile. Santiago-Chile.

Assessment of generic skills in communication and collaboration through an Objective Structured Clinical Examination in Emergency Radiology in a university residency program in Chile.

Resumen: Introducción: La Radiología de Urgencias es una unidad de aprendizaje longitudinal a toda la residencia. En ella los residentes se enfrentan a patología de urgencia en distintas modalidades de imagen. Además del dominio cognitivo, los residentes deben desarrollar habilidades de comunicación y colaboración que les permitan enfrentar adecuadamente los turnos de residencia. Previo a incorporarse a los turnos nocturnos, los residentes deben aprobar un Examen Clínico Objetivo Estructurado (ECOE). Propósito: Evaluar competencias de comunicación y colaboración a través de estaciones de ECOE de Radiología de Urgencias aplicado a residentes de $2^{\circ}$ año de residencia. Metodología: Se seleccionaron objetivos específicos a evaluar para las competencias de comunicación y colaboración y contenidos de Radiología Abdominal, Osteoarticular y Pediátrica para diseñar tres estaciones de ECOE, las cuales fueron incorporadas en el instrumento de evaluación aplicado el año 2017. Se calcularon porcentajes de logro por estación y tipo de pauta de evaluación. Para evaluar la implementación de las estaciones, se diseñó y aplicó una encuesta de percepción a los residentes. Resultados: Se diseñaron tres estaciones, dos para el rol Comunicador y una para el rol Colaborador. La validez de contenido se aseguró a través de un panel de expertos. Los porcentajes de logro global en las estaciones fluctuaron entre 74,7\% y 95,6\%. Las estaciones fueron bien evaluadas por parte de los residentes, quienes valoraron la incorporación de competencias diferentes al dominio cognitivo en el instrumento de evaluación. Conclusión: El ECOE permite certificar habilidades transversales como comunicación y colaboración de manera apropiada.

Palabras claves: Colaboración, Comunicación, Educación radiológica, Examen clínico objetivo estructurado, Radiología de urgencias.

Abstract: Introduction: Emergency radiology $(E R)$ is taught and learned throughout the entire residency. Trainees are exposed to different subspecialties ER cases and through varied imaging techniques. In addition to the "medical expert" domain, residents must develop communication and collaboration skills that will help them during their call schedule. Before they can take night-call, radiology residents must pass an Objective Structured Clinical Examination (OSCE). Purpose: To evaluate communication and collaboration competencies on an ER-OSCE applied to second-year radiology residents. Methods: Specific learning objectives were defined to evaluate communication and collaboration skills in abdominal, musculoskeletal and pediatric ER. Three OSCE stations were designed for this purpose and added to the evaluation instrument in 2017. On each station, resident performance was assessed with a specific template and percentage of goal achievement was calculated. Trainee's opinion of this new modality was obtained through a perception survey. Results: Three stations were designed, two for the communicator role and one for the collaborator competency. Validity of content confirmed through an expert panel. Percentages of goal achievement on each station varied between $74,7 \%$ and $96,6 \%$. The new stations were positively evaluated by residents, which valued that competencies other than "medical expert" were being 
assessed. Conclusion: ER-OSCE allows the appropriate assessment of generic competencies such as communication and collaboration in radiology.

Keywords: Collaboration, Communication, Emergency radiology, Objective structured clinical examination, Radiology education.

Garrido F., et al. Evaluación de habilidades transversales de comunicación y colaboración a través de un examen clínico objetivo estructurado de radiología de urgencias para residentes de un programa universitario de especialidad en radiología. Rev Chil Radiol 2019; 25(1): 35-41.

*Correo electrónico: Francisco Garrido Cisterna / fjgarridoc@uc.cl

Trabajo enviado el 31 de enero de 2019. Aceptado para publicación el 05 de febrero de 2019.

\section{Introducción}

La educación médica basada en competencias es un enfoque de formación de médicos para la práctica que está orientada hacia la adquisición de las habilidades finales del graduado, organizadas entorno a competencias derivadas del análisis de las necesidades de la sociedad y de los pacientes ${ }^{(1)}$. Es decir, las características del producto final (graduado) determinan el contenido curricular del programa de formación y su organización, los métodos y estrategias de enseñanza-aprendizaje, el proceso de evaluación, el clima educativo y la organización temporal del plan de estudios ${ }^{(2)}$.

La Dirección de Postgrado de la Escuela de Medicina de la Pontificia Universidad Católica de Chile adoptó el año 2010 el modelo de educación médica basada en competencias CanMEDS (Canadian Medical Education Directions for Specialists) del Royal College of Physicians and Surgeons of Canada (RCPSC) para sus programas de especialidades médicas. El modelo de competencias CanMEDS, desarrollado el año 1996 y revisado el 2005 y 2015, tiene como principal objetivo el articular una definición integral de las habilidades necesarias para todos los ámbitos de la práctica médica, entregando una base sólida para la educación médica ${ }^{(3)}$. Se enfoca en definir las habilidades necesarias que deben desarrollar todos los médicos para ejercer conforme a los requerimientos de sus pacientes y su familia, las comunidades y sociedad a la que sirven ${ }^{(4)}$. Este modelo de competencias comprende siete habilidades que representan los roles del médico: Médico Experto, Comunicador, Colaborador, Líder, Promotor de la Salud, Académico y Profesional(3), las cuales deben ser evaluadas durante la residencia para cumplir con los estándares del RCPSC.

Nuestro programa de especialidad en Radiología incorporó este modelo de competencias en la formulación de los programas de las distintas rotaciones de la residencia, además de definir las metodologías de aprendizaje e instrumentos de evaluación más adecuados para este propósito.

La radiología de urgencias es una unidad de aprendizaje longitudinal a toda la residencia, en la que los residentes se enfrentan a patología de urgencias a través de distintas modalidades de imagen. Las competencias CanMEDS para radiología de urgencias son los roles Comunicador, Colaborador, Líder y Profesional. Previo a la incorporación a los turnos nocturnos a partir del cuarto semestre de programa, los residentes deben aprobar un Examen Clínico Objetivo Estructurado (ECOE) que certifica las habilidades necesarias para asumir el rol de residente único supervisado(5).

El propósito de la presente intervención educacional es diseñar e incorporar nuevas estaciones al ECOE de Radiología de Urgencias que evalúen directa y explícitamente los roles CanMEDS Comunicador y Colaborador.

\section{Material y Métodos}

Para definir los objetivos específicos a evaluar, y con ello el contenido a incluir en cada estación, se creó un panel de jueces que consideró aspectos como la prevalencia de los problemas clínicos, urgencia de resolución y factibilidad de implementar en una estación de ECOE (validez de contenido). Los escenarios clínicos y las pautas de evaluación (que incluyen listas de cotejo, pautas de apreciación y/o rúbricas de desempeño global) se crearon de acuerdo a los formatos del Centro de Educación Médica y Ciencias de la Salud UC. Expertos en educación médica analizaron la factibilidad y coherencia de los escenarios clínicos creados. Lo anterior permitió la creación de las estaciones de ECOE, dos para comunicación y una para colaboración, cuyas características generales se resumen en la tabla 1.

Las estaciones se incorporaron en el ECOE de radiología de urgencias aplicado a los residentes de $2^{\circ}$ año en octubre de 2017. Los resultados obtenidos por los residentes fueron analizados considerando porcentaje de logro por estación y por tipo de pauta de evaluación.

Con el propósito de obtener una retroalimentación sobre la implementación y la experiencia de la intervención educacional por parte de los propios 
Tabla 1. Características generales de las estaciones de ECOE de Radiología de Urgencias.

\begin{tabular}{|c|c|c|c|}
\hline Rol CanMEDS & $\begin{array}{l}\text { Estación NN1 } \\
\text { Colaborador }\end{array}$ & $\begin{array}{l}\text { Estación №2 } \\
\text { Comunicador }\end{array}$ & $\begin{array}{l}\text { Estación N으 } \\
\text { Comunicador }\end{array}$ \\
\hline Competencia clave & $\begin{array}{l}\text { Trabajar efectivamente } \\
\text { con otros médicos y } \\
\text { profesionales de la } \\
\text { salud }\end{array}$ & $\begin{array}{l}\text { Redactar el informe } \\
\text { radiológico de } \\
\text { manera oportuna, } \\
\text { priorizando aquellos } \\
\text { exámenes que contengan } \\
\text { hallazgos críticos o que } \\
\text { requieran un manejo específico } \\
\text { o inmediato por parte de los } \\
\text { médicos de urgencia. }\end{array}$ & $\begin{array}{l}\text { Compartir con los pa- } \\
\text { cientes y sus } \\
\text { familias información } \\
\text { y planes respecto de } \\
\text { la atención de salud. }\end{array}$ \\
\hline Actividad a realizar & $\begin{array}{l}\text { Recomendar al } \\
\text { clínico un estudio de } \\
\text { imágenes por vía clínica } \\
\text { telefónica }\end{array}$ & $\begin{array}{l}\text { Producir un } \\
\text { informe radiológico }\end{array}$ & $\begin{array}{l}\text { Explicar a una } \\
\text { madre la condición } \\
\text { de su hijo. }\end{array}$ \\
\hline Contenido & $\begin{array}{l}\text { Radiología Abdominal - } \\
\text { Apendicitis Aguda }\end{array}$ & $\begin{array}{l}\text { Radiología Músculo- } \\
\text { esquelética - } \\
\text { Complicaciones de } \\
\text { una fractura costal. }\end{array}$ & $\begin{array}{l}\text { Radiología } \\
\text { Pediátrica - } \\
\text { Invaginación } \\
\text { intestinal. }\end{array}$ \\
\hline $\begin{array}{l}\text { Paciente } \\
\text { estandarizado }\end{array}$ & $\begin{array}{l}\text { Sí } \\
\text { (médico emergenciólogo) }\end{array}$ & No & Sí (madre) \\
\hline Pautas diseñadas & $\begin{array}{l}\text { Del alumno } \\
\text { Del evaluador } \\
\text { De evaluación del docente } \\
\text { De corrección del docente }\end{array}$ & $\begin{array}{l}\text { Del alumno } \\
\text { Hoja de respuestas del alumno } \\
\text { Del evaluador } \\
\text { De corrección del evaluador }\end{array}$ & $\begin{array}{l}\text { Del alumno } \\
\text { Guión del paciente } \\
\text { estandarizado } \\
\text { Del evaluador } \\
\text { De evaluación del PE } \\
\text { De evaluación al PE } \\
\text { De corrección del } \\
\text { evaluador } \\
\text { De corrección del PE }\end{array}$ \\
\hline \multicolumn{4}{|c|}{ Pautas de evaluación } \\
\hline $\begin{array}{l}\text { Lista de cotejo } \\
\text { Pauta de apreciación } \\
\text { Rúbrica }\end{array}$ & $\begin{array}{l}\text { Sí (12 ítems) } \\
\text { No } \\
\text { Sí (3 ítems) }\end{array}$ & $\begin{array}{l}\text { Sí (17 ítems) } \\
\text { No } \\
\text { No }\end{array}$ & $\begin{array}{l}\text { Sí (4 ítems) } \\
\text { Sí (16 ítems) } \\
\text { Sí (3 ítems) }\end{array}$ \\
\hline
\end{tabular}

residentes se diseñó una encuesta de percepción. Los resultados fueron analizados obteniendo medidas de estadística descriptiva y a través de la transcripción de los comentarios de los residentes.

La unidad de ética y seguridad de la investigación de la Facultad de Medicina UC aprobó la presente intervención educacional.

\section{Resultados}

Las tres estaciones creadas fueron incorporadas al examen de radiología de urgencias que en total comprendía 12 estaciones, cada una con un tiempo de 10 minutos, sin estaciones de descanso.

Los porcentajes de logro obtenidos exclusiva- mente del análisis de aquellos ítems que evalúan las competencias de Comunicación y Colaboración, éstos oscilan entre $75-100 \%$. Los porcentajes de logro para cada estación en general y por tipo de pauta de evaluación en particular se detallan en tabla 2.

La encuesta de percepción creada para ser contestada por los residentes se dividió en 2 partes. La primera parte de la encuesta fue contestada por vía electrónica por el $100 \%$ de los residentes. Los resultados de la primera parte de la encuesta de percepción, aplicada previo a la reunión de feedback sobre el desempeño individual, se presentan en las figuras 1 a 3 . La segunda parte de la encuesta recibió un $90 \%$ de respuestas. En ellas, se presentaban 
Tabla 2. Porcentajes de logro obtenidos por los residentes en las estaciones de ECOE.

\begin{tabular}{|c|c|c|c|c|c|c|}
\hline \multirow[t]{2}{*}{ Estación } & \multirow{2}{*}{$\begin{array}{l}\text { Porcentaje } \\
\text { global } \\
\text { de logro }\end{array}$} & \multirow{2}{*}{ Mínimo } & \multirow{2}{*}{ Máximo } & \multicolumn{3}{|c|}{ Por tipo de pauta de evaluación } \\
\hline & & & & \multirow{2}{*}{$\begin{array}{l}\text { Lista de } \\
\text { cotejo }\end{array}$} & \multirow{2}{*}{$\begin{array}{l}\text { Pauta de } \\
\text { apreciación }\end{array}$} & \multirow[t]{2}{*}{ Rúbrica } \\
\hline & & & & & & \\
\hline 1: Rol Colaborador & $88,3 \%$ & $62,5 \%$ & $100 \%$ & $92,5 \%$ & No aplica & $84,2 \%$ \\
\hline 2: Rol Comunicador & $74,7 \%$ & $41,2 \%$ & $88,2 \%$ & $74,7 \%$ & No aplica & No aplica \\
\hline 3: Rol Comunicador & $95,6 \%$ & $85,9 \%$ & $100 \%$ & $97,5 \%$ & $95,6 \%$ & $95 \%$ \\
\hline
\end{tabular}

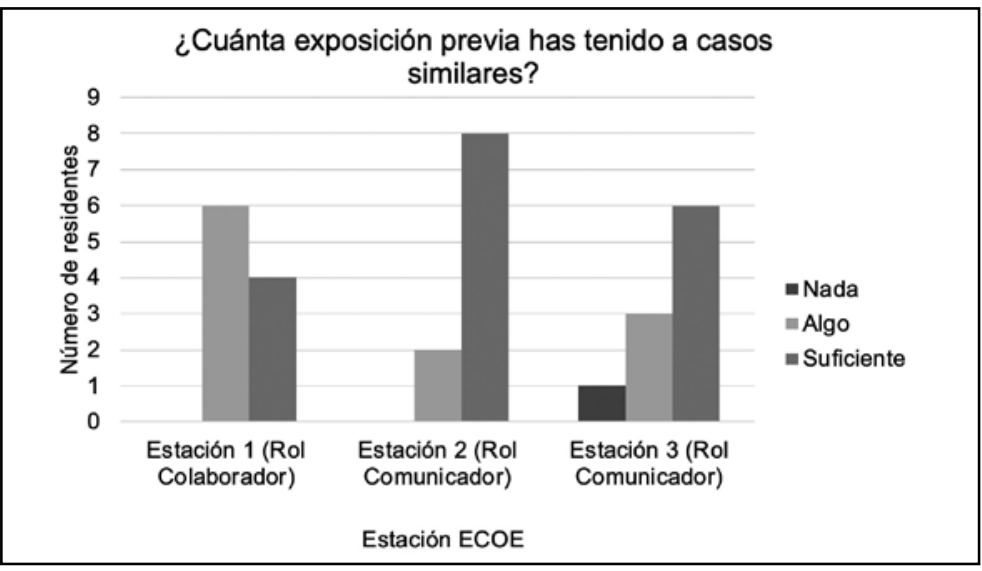

Figura 1. Resultados de la encuesta de percepción de residentes.

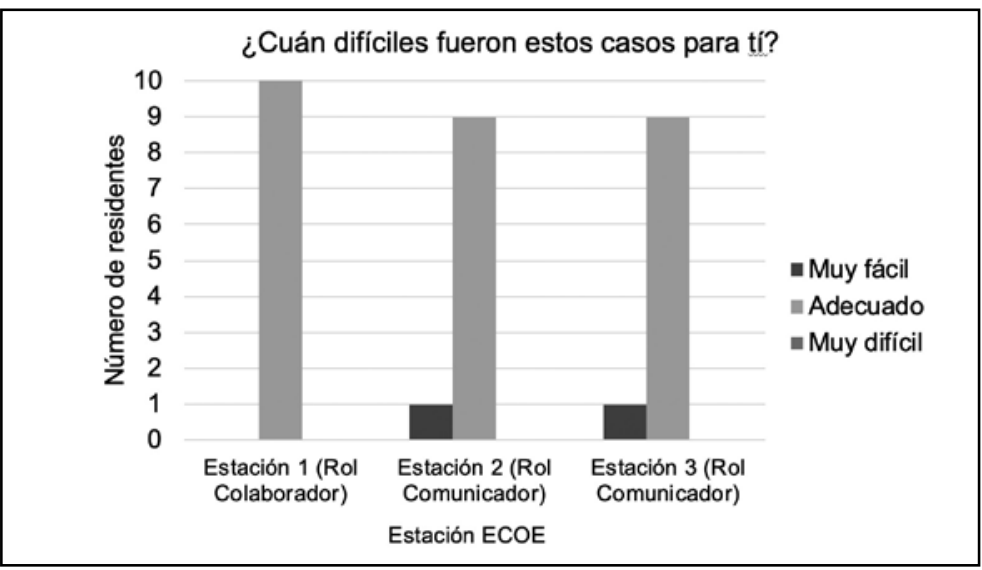

Figura 2. Resultados de la encuesta de percepción de residentes.

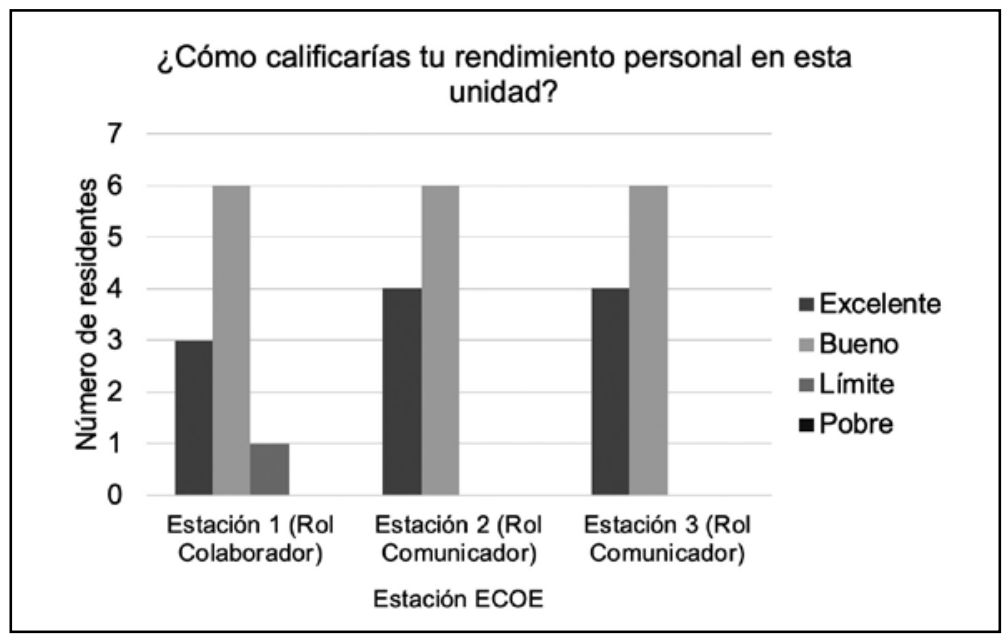

Figura 3. Resultados de la encuesta de percepción de residentes. 
algunas afirmaciones sobre el ECOE, en la que los residentes respondieron su grado de acuerdo con la afirmación utilizando una escala Likert de 1 a 4. En general existe un alto porcentaje de acuerdo con las afirmaciones presentadas, excepto en aquella relacionada con el tiempo destinado a las estaciones (Tabla 3).

Esta misma encuesta incluyó un espacio para comentarios abiertos sobre el examen. Se transcriben a continuación los comentarios más representativos:

"Valoro positivamente la inclusión de la estación de habilidades blandas: habilidad que en general no entrenamos, y que ya es conocido que muchos subespecialistas simplemente no tienen. Valoro el esfuerzo que significa organizar y realizar el ECOE por parte de los organizadores".

"Me parece buena idea la incorporación de habilidades blandas en el examen".

"Las situaciones creadas en las pruebas de habilidades blandas estuvieron bien hechas".

"Llamada telefónica creo que es muy adecuado que no involucre ver un examen en una estación, sino que contestar otras dudas".

"Los casos fueron adecuadamente revisados antes del OSCE, por lo que fueron atingentes a las habilidades evaluadas. Los tiempos fueron bien calculados".

Por otro lado, no se registraron comentarios sobre aspectos que deberían mejorar en una próxima versión, referidos a las nuevas estaciones de ECOE implementadas.

\section{Discusión}

Uno de los grandes avances en educación superior de las últimas décadas ha sido la educación basada en competencias. Sin embargo, establecer una definición de qué son las competencias ha sido objeto de amplio debate en la literatura educacional. Específicamente para educación médica, Epstein y Hundert definen las competencias como "el uso juicioso y habitual de la comunicación, conocimiento, habilidades técnicas, razonamiento clínico, emociones, valores y reflexión en la práctica diaria para el beneficio de los individuos y comunidades a las que se sirve"(6). Reflejan la relación entre las habilidades de las personas y la tarea que requiere que él o ella realice en un contexto particular en el mundo real, es decir, son cualidades personales y no acciones ${ }^{(7)}$.

Tabla 3. Porcentajes de acuerdo de los residentes con las afirmaciones sobre el ECOE de Radiología de Urgencias.

\begin{tabular}{|l|c|c|}
\hline En general (en) este ECOE... & $\begin{array}{c}\text { Muy de acuerdo + } \\
\text { De acuerdo }\end{array}$ & $\begin{array}{c}\text { En desacuerdo + } \\
\text { Muy en desacuerdo }\end{array}$ \\
\hline $\begin{array}{l}\text { Me ayudó a identificar mis fortalezas y } \\
\text { debilidades en Radiología de Urgencias }\end{array}$ & $100 \%$ & $0 \%$ \\
$\begin{array}{l}\text { Me estimuló a aprender más sobre algunos } \\
\text { de los tópicos cubiertos }\end{array}$ & $100 \%$ & $11,1 \%$ \\
Me enseñó algo nuevo & $88,9 \%$ & $11,1 \%$ \\
$\begin{array}{l}\text { Me entregó un feedback que fue valioso para mí } \\
\text { Evaluó mis habilidades con justicia }\end{array}$ & $88,9 \%$ & $22,2 \%$ \\
$\begin{array}{l}\text { Fue entretenido } \\
\text { Evaluó una amplia gama de competencias en }\end{array}$ & $77,8 \%$ & $33,3 \%$ \\
Radiología de Urgencias & $66,7 \%$ & $22,2 \%$ \\
$\begin{array}{l}\text { Fue un instrumento de evaluación que podría } \\
\text { repetir de nuevo }\end{array}$ & $77,8 \%$ & $22,2 \%$ \\
$\begin{array}{l}\text { El tiempo destinado a cada estación fue adecuado } \\
\text { La incorporación de evaluación de otras habilidades } \\
\text { (distintas al Médico Experto) es valioso dentro del } \\
\text { programa de residencia }\end{array}$ & $77,8 \%$ & $44,4 \%$ \\
\hline
\end{tabular}


Las competencias son multidimensionales, dinámicas, contextuales y desarrollables, involucrando múltiples dominios de habilidad desde el novato al maestro(8). En educación médica se han desarrollado varios perfiles de competencias, entre los que se encuentran el Tomorrow's Doctor (Reino Unido), Scottish Doctor (Escocia), Good Medical Practice (Australia), ACGME Outcomes Project (Estados Unidos) y CanMEDS (Canadá), siendo estos dos últimos los más difundidos en educación médica de residentes ${ }^{(9,10)}$.

El Examen Clínico Objetivo Estructurado, introducido por Ronald Harden en 1975 en la Universidad de Dundee (Escocia), es un instrumento de evaluación basado en los principios de objetividad y estandarización, en el cual los candidatos rotan a través de un circuito con una serie de estaciones con tiempo limitado, con el propósito de evaluar las competencias clínicas en un ambiente simulado(11).

El programa de especialidad en Radiología fue pionero en nuestra casa de estudios al introducir este instrumento de evaluación en educación médica de postgrado el año 2005, el cual tradicionalmente incluye casos clínicos con situaciones de urgencias en las áreas de radiografía de tórax, TAC de cuerpo, radiología osteoarticular, radiología pediátrica, ultrasonido-Doppler y neurorradiología. Las estaciones comprenden preguntas escritas de desarrollo corto en relación a descripción imagenológica del caso, diagnóstico más probable, diagnósticos diferenciales atingentes, tratamiento o conducta como residente a cargo del turno. Previo a esta intervención educacional, todas las estaciones del ECOE de Radiología de Urgencias han evaluado la competencia del Médico Experto, omitiendo otras competencias que, según la literatura, también son susceptibles de incorporar a este instrumento de evaluación como la comunicación y colaboración ${ }^{(14)}$.

En la presente intervención educacional se incorporaron nuevas estaciones al ECOE de radiología de urgencias como examen certificador de competencias adquiridas por los residentes previo a su incorporación a los turnos nocturnos como residente único supervisado. Éstas tres estaciones tuvieron como foco principal la evaluación de las competencias CanMEDS Comunicador y Colaborador, a través de situaciones de radiología de urgencias cotidianas, definidas por juicio de expertos, lo que amplía el espectro de competencias evaluadas durante la residencia, cumpliendo así con los estándares del RCSPC para la formación de especialistas médicos. Las tres estaciones fueron evaluadas con distintos tipos de pautas de evaluación (listas de cotejo, pautas de apreciación y rúbricas de desempeño global), aplicados por uno o más evaluadores por estación (docentes y paciente estandarizado), obteniendo porcentajes de logro similares entre sí en cada estación.

Si bien existen algunas experiencias de ECOE en radiología en la literatura educacional, estos son escasos y no están enfocados en radiología de urgencias $^{(15,16,17,18)}$, por lo que esta intervención educacional es relevante y novedosa.

Como fortaleza de esta experiencia educacional está la implementación del informe simulado, que se acerca a la realidad del trabajo de los residentes en las sesiones diarias a través de la redacción de informes de uno o más casos radiológicos en un tiempo determinado. Esto no solo simula la actividad más importante del médico radiólogo, sino que también permite evaluar otros aspectos relevantes como la comunicación escrita. Dentro de las limitaciones se reconoce que no se determinaron otras fuentes de validez de los resultados obtenidos en este instrumento de evaluación, diferentes a la validez de contenido. La simulación y el uso de paciente estandarizado no está incorporado dentro de las metodologías de enseñanza-aprendizaje del nuestro programa de especialidad en radiología, por lo que, en esta versión, los residentes se enfrentaron por primera vez a un paciente estandarizado en la evaluación misma. Esto debe ser corregido hacia el futuro a través de la simulación, debido a que no es posible evaluar a los residentes con un instrumento que incluya elementos desconocidos o que no hayan sido incorporados previamente durante el programa.

La evaluación de las competencias de los residentes es un desafío para los educadores médicos. Por el carácter profesionalizante de los programas de especialidades médicas es necesario asegurar la adquisición de competencias que permitan certificar que el especialista en formación será competente durante su desempeño profesional. En esta línea, la evaluación para el aprendizaje (assessment for learning) es un enfoque en que el programa de evaluación es usado para recolectar y combinar información desde varias fuentes para ilustrar acerca de las fortalezas y aspectos por mejorar de cada estudiante en forma individual, con el propósito de optimizar su aprendizaje $^{(19)}$. En este paradigma, la evaluación define la excelencia individualmente más que en relación con el grupo, es decir, fomenta la superación del estudiante comparándolo consigo mismo. La elección de los instrumentos de evaluación, como los ECOE, debe ser lo suficientemente flexible para adaptarse a las características particulares de los estudiantes.

El cambio de paradigma en la formación de médicos especialistas hacia una educación basada en competencias implica múltiples desafíos para los educadores médicos y docentes de los programas de residencia, entre ellos está la evaluación integral de las competencias de los residentes, más allá del dominio cognitivo.

\section{Referencias}

1. Frank JR, Mungroo R, Ahmad Y, Wang M, De Rossi S, 
Horsley T. Toward a definition of competency-based education in medicine: A systematic review of published definitions. Med Teach. 2010; 32(8): 631-637.

2. Harden RM. AMEE Guide No. 14: Outcome-based education: Part 1-An introduction to outcome-based education. Med Teach. 1999; 21(1): 7-14.

3. Frank JR, Snell L, Sherbino J E. CanMEDs 2015 Physician Competency Framework. CanMEDS 2015 Physician Competency Fram Ottawa R Coll Physicians Surg Canada [Internet]. 2015; 1-30. Disponible en: http:// www.royalcollege.ca/portal/page/portal/rc/canmeds/ resources/publications

4. Frank JR, Danoff D. The CanMEDS initiative: Implementing an outcomes-based framework of physician competencies. Med Teach. 2007; 29(7): 642-647.

5. Garrido $F$, Henríquez $H$, Cikutovic $P$, de Barbieri $F$, Burdiles Á, Riquelme C, et al. Resultados del examen clínico objetivo estructurado (ECOE) en el Programa de Postítulo en Radiología UC para evaluar competencias en radiología de urgencias. Investig en Educ Médica. 2017; 6(22): 129.

6. Epstein RM, Hundert EM. Defining and assessing professional competence. JAMA. 2002; 287(2): 226-235.

7. Ten Cate O, Scheele F. Competency-based postgraduate training: Can we bridge the gap between theory and clinical practice? Acad Med. 2007; 82(6): 542-547.

8. Taber S, Frank JR, Harris KA, Glasgow NJ, lobst W, Talbot M. Identifying the policy implications of competency-based education. Med Teach. 2010; 32(8): 687-691.

9. Lobst WF, Sherbino J, Cate O Ten, Richardson DL, Dath D, Swing SR, et al. Competency-based medical education in postgraduate medical education. Med Teach. 2010; 32(8): 651-656.

10. Kadmon M, Ten Cate O, Harendza S, Berberat P. Postgraduate Medical Education - an increasingly important focus of study and innovation. GMS J Med Educ. 2017; 34(5): Doc70.

11. Khan KZ, Ramachandran S, Gaunt K, Pushkar P. The Objective Structured Clinical Examination (OSCE): AMEE Guide No. 81. Part I: An historical and theoretical perspective. Med Teach. 2013; 35(9): e1437-146.

12. Harden R, Lilley $P$, Patricio $M$. The definitive guide to the OSCE: the Objective Structured Clinical Examination as a performance assessment. London: Elsevier Health Sciences; 2016.

13. Shumway JM, Harden RM, Association for Medical Education in Europe. AMEE Guide No. 25: The assessment of learning outcomes for the competent and reflective physician. Med Teach. 2003; 25(6): 569-584.

14. Bandiera G, Sherbino J. The CanMEDS assessment tools handbook [Internet]. 2006. Disponible en: http:// www.royalcollege.ca/CanMEDStoolsguide

15. Probyn L, Lang C, Finlay K, Herold J, Bartlett E, Glover Takahashi S. Assessment of the Intrinsic CanMEDS Roles in Diagnostic Radiology Residents using an Objective Structured Clinical Assessment (OSCE). MedEdPublish. 2016; 5(2).

16. Nadeem N, Zafar AM, Zuberi RW, Ahmad MN. Faculty and patient evaluations of radiology residents' communication and interpersonal skills. J Pak Med Assoc. 2012; 62(9): 915-919.

17. Lown BA, Sasson JP, Hinrichs P. Patients as Partners in Radiology Education. Acad Radiol. 2008; 15(4): 425-432.

18. Williamson KB, Steele JL, Gunderman RB, Wilkin TD, Tarver RD, Jackson VP, et al. Assessing Radiology Resident Reporting Skills. Radiology. 2002; 225(3): 719-722.

19. Schuwirth LWT, Van der Vleuten CPM. Programmatic assessment: From assessment of learning to assessment for learning. Med Teach. 2011; 33(6): 478-485. 